\title{
Complications and Outcomes of Surgical Patients Operated During COVID 19: a Single Centre Study
}

\author{
Surender Verma ${ }^{1}$ (i) $\cdot$ Pradeep Garg $^{1} \cdot$ Mridul Gera $^{1} \cdot$ Shivika Agarwal ${ }^{1} \cdot$ Anjali Verma $^{2}$
}

Received: 16 December 2021 / Accepted: 26 January 2022/ Published online: 1 February 2022

(c) Association of Surgeons of India 2022

COVID-19 pandemic has been widespread and devastating and has created unprecedented challenges for the whole medical fraternity. Mainly emergency surgeries were carried out during the pandemic, and data on postoperative outcomes of COVID-19 patients is limited [1, 2]. Hence, this study was planned to find out post-operative outcome of RTPCR-positive COVID-19 patients who underwent emergency surgeries in a tertiary care institute. Retrospective data of those patients which included various parameters such as age, sex, COVID-19-related symptoms, comorbidities, surgical procedure done, length of hospital stay, postoperative complications, ICU admissions, and final outcome was collected and analysed.

Twenty-three RT-PCR-positive patients who underwent emergency surgery during first and second wave of COVID-19 (1 March 2020 to 30 June 2021) were included in the study. A total of 6 and 17 were operated during 1st and 2nd wave of COVID-19, respectively. M:F ratio was 4:1 and mean age of the patients was 47 years. Before surgery, 18 were symptomatic for COVID-19, and 5 were asymptomatic. Fever was the main clinical symptom in 15 patients $(65.2 \%)$, cough in 10 patients $(43.4 \%)$, and dyspnoea in 3 patients (13\%). Two patients were diabetic, 7 patients were hypertensive, and 2 patients had taken ATT in the past for pulmonary Koch's, whereas no comorbidities were recorded in 12 patients $(52.1 \%)$. Fourteen patients underwent surgery for perforation peritonitis, 6 for acute appendicitis, 2 for blunt trauma abdomen, and 1 for absolute dysphagia. Seventeen patients were operated under general anaesthesia and 6 under spinal anaesthesia. Fourteen $(60.8 \%)$ patients had postoperative complications among which 11 developed pulmonary complications, 2 had burst abdomen, and

Surender Verma

drsurn@gmail.com

1 Department of General Surgery, PGIMS, 117- A, Sector 14, Rohtak, India

2 Department of Pediatrics, PGIMS, Rohtak, India
1 had gut gangrene for which the patient was reoperated. Mean duration of hospital stay was 9.6 days, 15 (65.2\%) patients required intensive care unit (ICU) care where they were given anticoagulants and steroids, and out of those, 9 (39.1\%) patients expired postoperatively.

In our study, it was observed that postoperative morbidity and mortality were high in COVID-19-positive patients. Several articles had already reported negative outcomes of surgery in infected patients since the beginning of the pandemic [2-4]. An international, multicentre, cohort study had found that postoperative pulmonary complications occur in half of patients with perioperative SARS-CoV-2 infection and were associated with high mortality (23.8\%) [3]. Findings from a multicentre Canadian cohort study and a Chinese study suggested that COVID-19 patients undergoing a surgery had mortality rate of $20.5 \%$ with $44.1 \%$ requiring ICU admissions and $15.9 \%$ with $27 \%$ ICU admissions respectively $[1,4]$.

Another Italian multicentre study recorded 32\% intensive care unit admissions, $33.8 \%$ postoperative pulmonary complications, and $14.7 \%$ postoperative deaths [5]. Our study had more ICU admissions and high mortality as compared to the previous studies as ours was a single centre study involving a smaller number of patients requiring emergency surgeries and the patients who were operated were already sick. Pulmonary complications were the most reported complications and were responsible for morbidity and mortality in previous studies, and same findings were there in our study [2]. As maximum patients succumbed due to pulmonary complications, COVID-19 infection was the main contributory factor for this catastrophic outcome. Hence, it is important to adopt strategies like specialised ICU care and chest physiotherapy that will reduce pulmonary complications post operatively.

Despite high morbidity and mortality, most of studies have concluded that emergency surgery should not be postponed. Moreover, such studies will help to formulate policies for better post-operative care in COVID-19-positive 
patients. In addition, multiple studies involving many centres will be helpful to reach a definitive conclusion regarding outcome of these patients.

Author Contribution SV: implemented the study, analysis, and wrote manuscript; PG, AV: supervised data collection and provided inputs in manuscript; MG, SA: collected data and contributed to writing of manuscript.

\section{Declarations}

Conflict of Interest The authors declare no competing interests.

\section{References}

1. Carrier FM, Amzallag É, Lecluyse V, Côté G, Couture ÉJ, D'Aragon F et al (2021) Postoperative outcomes in surgical COVID-19 patients: a multicenter cohort study. BMC Anesthesiol 21(1):15. https://doi.org/10.1186/s12871-021-01233-9
2. Doglietto F, Vezzoli M, Gheza F, Lussardi GL, Domenicucci M, Vecchiarelli L et al (2020) Factors associated with surgical mortality and complications among patients with and without coronavirus Disease 2019 (COVID-19) in Italy. JAMA Surg 155(8):1-14

3. COVIDSurg Collaborative (2020) Mortality and pulmonary complications in patients undergoing surgery with perioperative SARS-CoV-2 infection: an international cohort study. Lancet 396(10243):27-38

4. Lei S, Jiang F, Su W, Chen C, Chen J, Mei W et al (2020) Clinical characteristics and outcomes of patients undergoing surgeries during the incubation period of COVID-19 infection. EClinicalMedicine 21:100331

5. De Luca M, Sartori A, Vitiello A, Piatto G, Noaro G, Olmi S et al (2021) Complications and mortality in a cohort of patients undergoing emergency and elective surgery with perioperative SARSCoV-2 infection: an Italian multicenter study. Teachings of Phase 1 to be brought in Phase 2 pandemic. Updates Surg 73(2):745-52. https://doi.org/10.1007/s13304-020-00909-0

Publisher's Note Springer Nature remains neutral with regard to jurisdictional claims in published maps and institutional affiliations. 